\title{
Prognostic significance of enlarged paraaortic lymph nodes detected during left-sided colorectal cancer surgery: a single-center retrospective cohort study
}

\author{
Jaram Lee, Hyeong-min Park, Soo Young Lee ${ }^{*}$ (D), Chang Hyun Kim and Hyeong Rok Kim
}

\begin{abstract}
Background: Very few studies have been conducted on the treatment strategy for enlarged paraaortic lymph nodes (PALNs) incidentally detected during surgery. The purpose of this study was to investigate the benefit of lymph node dissection in patients with incidentally detected enlarged PALNs.

Methods: We retrospectively reviewed patients with left colon and rectal cancer who underwent surgical resection with PALN dissection between January 2010 and December 2018. The predictive factors for pathologic PALN metastasis (PALNM) were analyzed, and survival analyses were conducted to identify prognostic factors.

Results: Among 263 patients included, 19 (7.2\%) showed pathologic PALNM and 5 (26.33\%) had enlarged PALNs incidentally detected during surgery. These 5 patients accounted for $2.2 \%$ of 227 patients who had no evidence of PALNM on preoperative radiologic examination. Radiologic PALNM (odds ratio [OR] 12.737, 95\% confidence interval [CI] 3.472-46.723) and radiologic distant metastasis other than PALNM (OR $=4.090,95 \% \mathrm{Cl} 1.011-16.539)$ were independent predictive factors for pathologic PALNM. Pathologic T4 stage (hazard ratio [HR] 2.196, 95\% Cl 1.0634.538) and R2 resection ( $H R$ 4.643, 95\% Cl 2.046-10.534) were independent prognostic factors for overall survival (OS). In patients undergoing RO resection, pathologic PALNM was not associated with 5 -year OS (90\% vs. 82.2\%, $p=$ 0.896).

Conclusion: Dissection of enlarged PALNs incidentally detected during colorectal surgery may benefit patients with favorable survival outcomes.
\end{abstract}

Keywords: Colorectal cancer, Paraaortic lymph node metastasis, Paraaortic lymph node dissection

\section{Introduction}

Colorectal cancer is the third most common cancer in men and the second most common cancer in women, with 1.8 million new cases in 2018 according to the World Health Organization GLOBOCAN database [1]. Approximately $22 \%$ of patients with colorectal cancer

\footnotetext{
*Correspondence: syleecrs@gmail.com

Department of Surgery, Chonnam National University Hwasun Hospital and Medical School, 322 Seoyang-ro Hwasun-eup, Hwasun-gun, Jeonnam 58128, South Korea
}

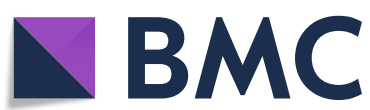

are diagnosed at stage IV. The 5-year relative survival of colorectal cancer patients of all stages is approximately $67 \%$, whereas the 5 -year relative survival of stage IV patients is only $15 \%$ [2].

According to the American Joint Committee on Cancer (AJCC) classification, paraaortic lymph node (PALN) metastasis (PALNM) is categorized as distant metastasis (M1), and therefore stage IV [3]. PALNM occurs in approximately $2 \%$ of colorectal cancer patients [4]. Previous studies have shown that patients with PALNM have

(c) The Author(s). 2021 Open Access This article is licensed under a Creative Commons Attribution 4.0 International License, which permits use, sharing, adaptation, distribution and reproduction in any medium or format, as long as you give appropriate credit to the original author(s) and the source, provide a link to the Creative Commons licence, and indicate if changes were made. The images or other third party material in this article are included in the article's Creative Commons licence, unless indicated otherwise in a credit line to the material. If material is not included in the article's Creative Commons licence and your intended use is not permitted by statutory regulation or exceeds the permitted use, you will need to obtain permission directly from the copyright holder. To view a copy of this licence, visit http://creativecommons.org/licenses/by/4.0/ The Creative Commons Public Domain Dedication waiver (http://creativecommons.org/publicdomain/zero/1.0/) applies to the data made available in this article, unless otherwise stated in a credit line to the data. 
a significantly lower survival rate than those without PALNM [5-8]. Although several studies have shown that PALN dissection (PALND) improves survival in patients with PALNM, there has been no clearly defined standard treatment for PALNM. Moreover, very few studies have been conducted on the treatment strategy for enlarged PALNs incidentally detected during colorectal cancer surgery.

Therefore, we designed this study to investigate the probability of pathologic PALNM and the benefit of PALND in patients with enlarged PALNs incidentally detected during colorectal cancer surgery.

\section{Methods}

We retrospectively reviewed patients with primary colorectal cancer who underwent surgical resection at our institution between January 2010 and December 2018. This study was approved by the institutional review board of our institution (CNUHH-2020-157).

Patients with left-sided colon and rectal cancer who underwent colorectal surgery with PALND were included in this study. We included all patients with retrieved PALNs regardless of whether or not PALNM was suspected on preoperative radiologic examination. Patients without suspected PALNM in the preoperative imaging study were classified into the incidentally detected PALN group.

The clinicopathologic factors of the included patients were retrospectively collected through a review of medical records, which included demographic data, preoperative evaluation, operative findings, pathologic characteristics, and postoperative outcomes. Preoperative evaluation included serum levels of carcinoembryonic antigen (CEA), colonoscopy, abdominopelvic computed tomography (CT), and rectal magnetic resonance imaging. Positron emission tomography (PET) was selectively performed when distant metastasis was suspected. PALNM was suspected when the PALN was $>8$ $\mathrm{mm}$ or $>5 \mathrm{~mm}$ and showed heterogeneity and an irregular outer border on abdominopelvic CT scan. In PET$\mathrm{CT}$, the presence of PALNs with higher fluorodeoxyglucose uptake than adjacent normal organs was considered to indicate PALNM.

All patients underwent mechanical bowel preparation before surgery. Second-generation cephalosporin was routinely administered within $1 \mathrm{~h}$ before surgery as an empirical antibiotics. In most cases, D3 dissection with high ligation of the inferior mesenteric artery was performed. For tumors below the peritoneal reflection, total mesorectal excision was performed. The detailed surgical technique has been previously described [9].

The extent of PALND was determined at the surgeon's discretion. In cases of highly suspicious PALNM, the PALNs were dissected from the aortic bifurcation to the lower border of the renal vein. In some cases with a single suspicious metastatic PALN, only the suspicious PALN was dissected. In most cases, the surgical procedure including PALND was performed using a laparoscopic approach.

R0 resection was defined as no macroscopic or microscopic residual tumor after surgery, and with no remnant tumor observed on follow-up radiologic examination. R2 resection was defined as a gross residual tumor remaining after surgery or a remnant tumor observed on the first follow-up CT. Histopathologic examination was performed by a specialized pathologist according to standard procedures. The classification system of the AJCC (seventh edition) was used to determine the pathologic stage. Adjuvant chemotherapy was recommended for advanced colorectal cancer, after considering the patient's general condition. Follow-up examinations included chest $\mathrm{CT}$, abdominopelvic $\mathrm{CT}$, and serum CEA test every 6 months and colonoscopy every 2 years. Recurrence was defined as recurrent disease on clinical, radiologic, or pathologic examination. Overall survival (OS) was defined as the period from surgery to death from any cause.

Categorical variables were compared using the $\chi^{2}$ test or Fisher's exact test, and continuous variables were compared using Student's $t$ test. Logistic regression analysis was used to investigate the predictive factors for PALNM. Survival outcomes were compared using the Kaplan-Meier method and log-rank test. Multivariate analysis with a Cox proportional hazard model was used to identify prognostic factors for OS. Variables that were significant at $p<0.10$ in the univariate analysis were entered into a multivariate model. Statistical significance was set at $p<0.05$. All statistical analyses were performed using IBM SPSS version 23 (IBM, Armonk, NY).

\section{Results}

A total of 263 patients with left colon and rectal cancer who underwent PALND were included, and 19 (7.2\%) of them showed pathologic PALNM. Of these 19 patients, 14 (73.7\%) were suspected to have PALNM on preoperative radiologic examination, and $5(26.3 \%)$ had enlarged PALNs incidentally detected during surgery. These 5 patients accounted for $2.2 \%$ of the 227 patients who had no evidence of PALNM on preoperative radiologic examination (Fig. 1). Frozen-section biopsy for PALNs was performed in $29(11.0 \%)$ of the 263 included patients. Of the 29 patients, 5 (17.2\%) had metastatic adenocarcinoma in frozen-section analysis, 4 of whom had suspicious PALNM on preoperative imaging study.

Table 1 shows the clinicopathological characteristics according to pathologic PALNM. Regarding preoperative characteristics, higher clinical T stage (cT4 29.0\% vs. cT1 0.0\%, cT2 3.2\%, cT3 3.8\%; $p<0.001$ ), higher clinical 


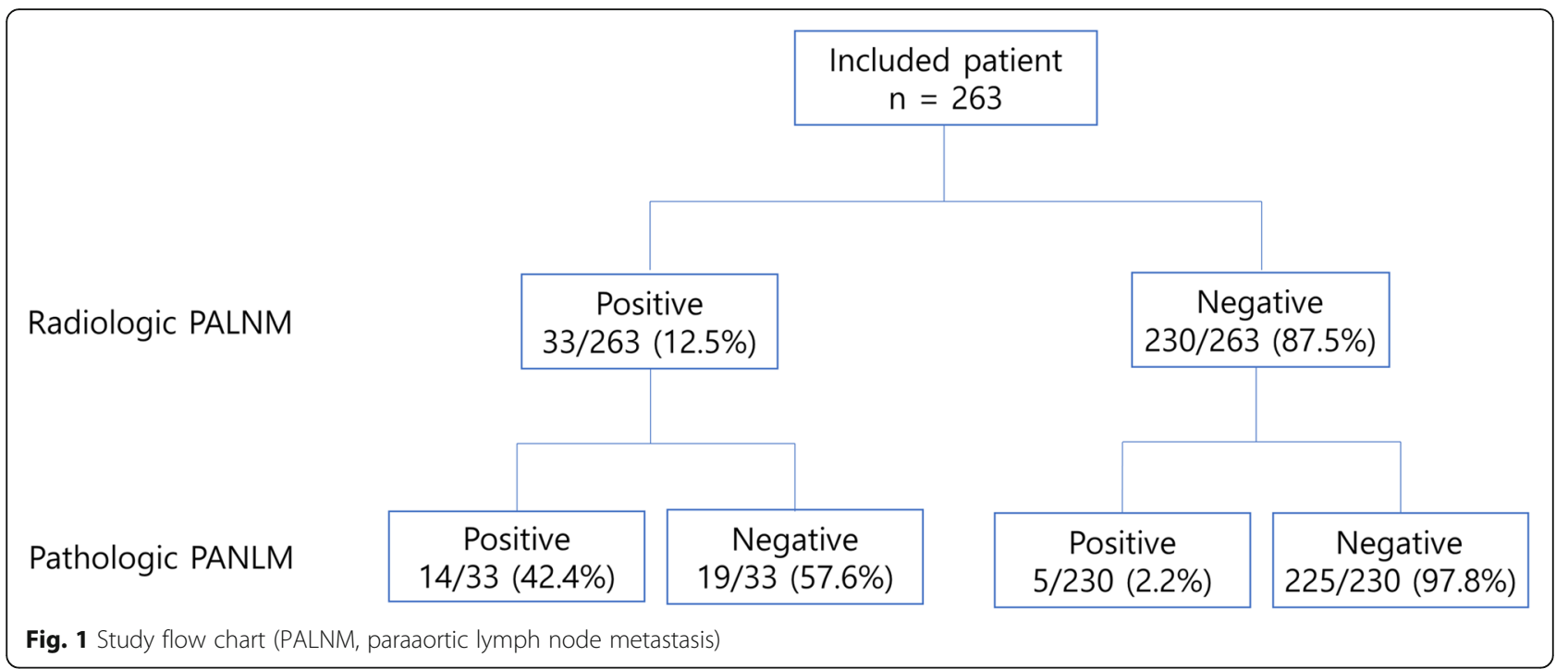

$\mathrm{N}$ stage (cN2 17.6\%, vs. cN0 2.1\%, cN1 1.3\%; $p<0.001$ ), radiologic PALNM (42.4\% vs. $2.2 \%, p<0.001)$, and radiologic distant metastasis other than PALNM (31.8\% vs. $5.0 \%, p<0.001)$ were associated with a higher incidence of pathologic PALNM. Regarding pathologic characteristics, larger tumor size $(>5 \mathrm{~cm})$, lymphovascular invasion and poor differentiation were associated with pathologic PALNM.

In multivariate analysis, radiologic PALNM (odds ratio [OR] 12.737, 95\% confidence interval [CI] 3.472-46.723, $p<0.001)$ and radiologic distant metastasis other than PALNM (OR 4.090; 95\% CI, 1.011-16.539; $p=0.048$ ) were independent predictive factors for pathologic PALNM. However, higher clinical $\mathrm{T}$ stage and $\mathrm{N}$ stage were not predictive factors for pathologic PALNM in the multivariate analysis (Table 2).

Table 3 shows postoperative complications. The overall 30 -day morbidity rate was $18.2 \%(48 / 263)$, and the most common complication was prolonged postoperative ileus (6.8\%). Anastomotic leakage occurred in 4.2\% $(11 / 263)$ of all the included patients. No postoperative mortality was recorded.

Prognostic factors for OS are described in Table 4. In univariate survival analysis, pathologic $\mathrm{T} 4$ stage $(37.3 \%$ vs. $82.1 \%, p<0.001)$, pathologic N1-2 stage $(65.8 \%$ vs. $87.7 \%, p=0.001), \quad$ M1 stage $(38.8 \%$ vs.81.5\%, $p<0.001)$, pathologic PALNM $(54.0 \%$ vs. $78.6 \%, p=0.002)$, and $\mathrm{R} 2$ resection $(12.8 \%$ vs. $82.3 \%$, $p<0.001$ ) were associated with poorer OS (Table 4 , Fig. 2a). Because $M$ stage and R0 resection were highly correlated with each other (correlation coefficient $=0.727, p<0.001$ ), $\mathrm{M}$ stage was excluded from the multivariable survival analysis. In the multivariable analysis, pathologic T4 stage (hazard ratio [HR] 2.196, 95\% CI 1.063-4.538, $p=0.034$ ) and R0 resection (HR
4.643, 95\% CI $2.046-10.534, p<0.001)$ were independent prognostic factors for OS.

Among all included patients, 20 (7.6\%) underwent R2 resection. Of 19 patients with pathologic PALNM, 9 patients (47.4\%) underwent R0 resection and 10 patients (52.6\%) underwent R2 resection. When comparing the survival outcome according to the radicality of resection in patients with PALNM, OS was significantly higher in patients undergoing R0 resection (5-year OS, $90.0 \%$ vs. $0.0 \% ; p=0.014$ ) (Fig. 2b). In the subgroup analysis of patients who underwent R0 resection, no difference in 5year OS $(90.0 \%$ vs. $82.2 \%, p=0.896)$ was found between patients with and without pathologic PALNM (Fig. 2c).

\section{Discussion}

In the present study, we investigated the necessity of PALND in patients with incidentally detected enlarged PALNs. The results showed that even in patients with no evidence of PALNM on preoperative radiologic examination, pathologic PALNM was identified in a small but non-negligible proportion (2.2\%). Patients with pathologic PALNM showed poorer OS than those without pathologic PALNM. However, when R0 resection was achieved, patients with pathologic PALNM had comparable OS to those without pathologic PALNM.

PALNM is a known poor prognostic factor in patients with colorectal cancer [4-7]. In cases of liver or lung metastasis, radical resection of the metastatic lesion is known to be beneficial for survival [10-12]. However, there is no established standard treatment for PALNM, which is relatively rarer than liver or lung metastasis [7, $8,13]$. Recently, several studies have reported that PALN D increased survival in patients with PALNM $[6,7,13-$ 15]. For example, Choi et al. reported that the 5-year OS of the PALND group was significantly higher than that 
Table 1 Characteristics according to pathologic PALNM

\begin{tabular}{|c|c|c|c|c|}
\hline Characteristics & & Pathologic PALNM $(-)(n=244)$ & Pathologic PALNM $(+)(n=19)$ & $p$ \\
\hline \multicolumn{5}{|l|}{ Preoperative } \\
\hline \multirow[t]{2}{*}{ Sex } & Male & $158(94.0 \%)$ & $10(6.0 \%)$ & 0.325 \\
\hline & Female & $86(90.5 \%)$ & $9(9.5 \%)$ & \\
\hline Age (years) & & $63.61 \pm 11.846$ & $63.37 \pm 10.761$ & 0.931 \\
\hline $\mathrm{BMI}\left(\mathrm{kg} / \mathrm{m}^{2}\right)$ & & $22.2 \pm 2.7$ & $22.2 \pm 2.6$ & 0.929 \\
\hline \multirow[t]{2}{*}{ ASA } & 1,2 & $224(92.9 \%)$ & $17(7.1 \%)$ & 0.665 \\
\hline & 3,4 & $20(90.9 \%)$ & $2(9.1 \%)$ & \\
\hline Preoperative & $<5$ & $160(94.7 \%)$ & $9(5.3 \%)$ & 0.133 \\
\hline CEA (ng/mL) & $\geq 5$ & $82(89.1 \%)$ & $10(10.9 \%)$ & \\
\hline \multirow[t]{4}{*}{ Clinical T stage } & cT1 & $13(100 \%)$ & $0(0.0 \%)$ & $<0.001$ \\
\hline & cT2 & $30(96.8 \%)$ & $1(3.2 \%)$ & \\
\hline & cT3 & $179(95.2 \%)$ & $9(4.8 \%)$ & \\
\hline & cT4 & $22(71.0 \%)$ & $9(29.0 \%)$ & \\
\hline \multirow[t]{3}{*}{ Clinical N stage } & cNO & 94 (97.9\%) & $2(2.1 \%)$ & $<0.001$ \\
\hline & $\mathrm{cN1}$ & 75 (98.7\%) & $1(1.3 \%)$ & \\
\hline & $\mathrm{cN} 2$ & 75 (82.4\%) & $16(17.6 \%)$ & \\
\hline Radiologic & Negative & $225(97.8 \%)$ & $5(2.2 \%)$ & $<0.001$ \\
\hline PALNM & Positive & 19 (57.6\%) & $14(42.4 \%)$ & \\
\hline Radiologic DM & Negative & $229(95.0 \%)$ & $12(5.0 \%)$ & $<0.001$ \\
\hline Other than PALNM & Positive & 15 (68.2\%) & $7(31.8 \%)$ & \\
\hline \multirow[t]{2}{*}{ Tumor location } & Colon & $107(93.0 \%)$ & $8(7.0 \%)$ & 0.882 \\
\hline & Rectum & $137(92.6 \%)$ & $11(7.4 \%)$ & \\
\hline \multicolumn{5}{|l|}{ Operative } \\
\hline \multirow[t]{2}{*}{ Surgical procedure } & Open & $6(60.0 \%)$ & $4(40.0 \%)$ & 0.003 \\
\hline & Laparoscopy & $238(94.1 \%)$ & $15(5.9 \%)$ & \\
\hline \multirow[t]{2}{*}{ Operative time (min) } & $<180$ & $197(93.8 \%)$ & $13(6.2 \%)$ & 0.232 \\
\hline & $\geq 180$ & 47 (88.7\%) & $6(11.3 \%)$ & \\
\hline \multicolumn{5}{|l|}{ Pathologic } \\
\hline \multirow[t]{2}{*}{ Tumor size (cm) } & $<5$ & $130(96.3 \%)$ & $5(3.7 \%)$ & 0.03 \\
\hline & $\geq 5$ & $113(89.0 \%)$ & $14(11.0 \%)$ & \\
\hline Lymphovascular & Negative & 203 (97.6\%) & $5(2.4 \%)$ & $<0.001$ \\
\hline Invasion & Positive & 41 (74.5\%) & $14(25.5 \%)$ & \\
\hline Perineural & Negative & $148(94.9 \%)$ & $8(5.1 \%)$ & 0.146 \\
\hline Invasion & Positive & $96(89.7 \%)$ & $11(10.3 \%)$ & \\
\hline \multirow[t]{2}{*}{ Differentiation } & $w / d, m / d$ & $227(93.4 \%)$ & $16(6.6 \%)$ & 0.031 \\
\hline & $\mathrm{p} / \mathrm{d}$ & 7 (70.0\%) & $3(30.0 \%)$ & \\
\hline
\end{tabular}

Data are presented as mean \pm standard deviation or number (percentage)

PALNM Paraaortic lymph node metastasis; BMI Body mass index; ASA American Society of Anesthesiologists; CEA Carcinoembryonic antigen; DM Distant metastasis; $w / d$ Well differentiated; $m / d$ Moderately differentiated; $p / d$ Poorly differentiated

of the control group (53.4\% vs. $12 \%, p=0.045$ ) [6]. Ogura et al. reported that the 5-year cancer-specific survival was significantly higher in the R0 resection group than in the control group with palliative resection in patients with extra-regional lymph node metastasis [16]. Gagniere et al. reported the outcome of radical retroperitoneal lymphadenectomy in patients with retroperitoneal nodal metastases from colorectal cancer [17]. They suggested that the 5-year OS of patients who underwent lymphadenectomy was significantly higher than that of patients treated with non-surgical treatment. However, some authors have reported that PALND is 
Table 2 Multivariate analysis of predictive factors for pathologic PALNM

\begin{tabular}{lll}
\hline Variables & Pathologic PALNM & $\boldsymbol{p}$ \\
\cline { 2 - 3 } & OR (95\% Cl) & 0.081 \\
\hline Clinical T stage (cT4 vs. CT1-3) & $3.182(0.865-11.701)$ & 0.207 \\
Clinical N stage (cN2 vs. CN0-1) & $2.705(0.577-12.671)$ & $<0.001$ \\
Radiologic PALNM (positive vs. negative) & $12.737(3.472-46.723)$ & 0.048 \\
Radiologic DM other than PALNM (positive vs. negative) & $4.090(1.011-16.539)$ & \\
\hline
\end{tabular}

PALNM Paraaortic lymph node metastasis; OR Odds ratio; Cl Confidence interval; DM Distant metastasis

not recommended because of its technical difficulties and lack of survival benefit [18]. Others reported a high recurrence rate after PALND even when performed in selective patients with PALNM [19].

Determining the presence of PALNM through a preoperative imaging study before surgery and establishing an appropriate surgical plan are essential. In this study, radiologic PALNM was identified as a predictive factor for pathologic PALNM in the multivariable analysis. Nakai et al. evaluated the diagnostic value of CT and $\mathrm{PET} / \mathrm{CT}$ in predicting PALNM [20]. When the diagnosis was based on CT combined with PET/CT, the diagnostic ability of PET/CT was $93.8 \%$ in patients who had no predictive CT findings. However, in patients with a suspected lesion on CT, the diagnostic ability of PET/CT was decreased to $70.6 \%$. According to a study by Wong et al., among 264 patients suspected of having PALNM on radiologic examination, 118 patients showed positive PALNM in pathology, and the positive predictive value was only $44.7 \%$ [5]. Similarly, in the present study, the accuracy of preoperative imaging in predicting PALNM was $89.7 \%$, whereas the positive predictive value was only $42.4 \%$. Therefore, it is not easy to accurately diagnose PALNM based on preoperative imaging findings.

Surgeons often incidentally detect enlarged PALNs during surgery and deliberate about whether PALND should be performed. However, no standard treatment has been established for the management of incidentally detected enlarged PALNs in colorectal cancer patients. Importantly, in the present study, there were five (2.2\%) false-negative cases in which PALNM was not detected

Table 3 Postoperative complications

\begin{tabular}{ll}
\hline Morbidity & $\boldsymbol{n}(\%)$ \\
\hline Total & $48(18.2 \%)$ \\
Bleeding & $2(0.8 \%)$ \\
lleus & $18(6.8 \%)$ \\
Anastomotic leakage & $11(4.2 \%)$ \\
Urinary dysfunction & $13(4.9 \%)$ \\
Pneumonia & $1(0.4 \%)$ \\
Wound infection & $6(2.3 \%)$ \\
Deep SSI & $5(1.9 \%)$ \\
\hline
\end{tabular}

SSI Surgical site infection on preoperative imaging but PALND was performed incidentally. In addition, a significant difference in OS was observed between the R0 and R2 resection groups (90.0\% vs. $0.0 \%, p=0.014)$. When R0 resection was achieved in patients with PALNM their OS was comparable to that of patients without PALNM $(90.0 \%$ vs. $82.2 \%, p=0.896$ ). Therefore, we recommend performing PALND not only in patients with radiologically suspected PALNM but also in patients with enlarged PALNs incidentally detected during surgery, especially in patients with radiologic distant metastasis other than PALNM.

Frozen-section biopsy of an enlarged PALN may be helpful to decide whether to proceed with further PALN D. In the present study, frozen-section biopsy was performed in only $11.0 \%$ (29/263) of patients, and 5 (17.2\%) of them showed PALNM. Because the present study was retrospective, it was impossible to investigate whether the extent of surgery changed according to the result of frozen-section biopsy. However, given the fact that PALNM had not been found in 24 (83.8\%) of the 29 patients with frozen-section biopsy, frozen-section biopsy may be helpful to avoid unnecessary wider PALND, preventing possible long-term complications.

Several studies have reported that retroperitoneal lymph node dissection is related to sexual dysfunction due to hypogastric nerve injury, chylous ascites, and lymphoceles [21, 22]. According to a systematic review by Wong et al. [5], the postoperative complication rate of PALND ranged from 7.8 to $33 \%$. We observed a postoperative complication rate of $18.2 \%$, with no postoperative mortality. The morbidity rate in the present study was comparable to that in our previous studies (13.9$31.2 \%$ ) on rectal cancer surgery [23, 24]. PALND can be safely performed with minimal additional surgical complications.

This study had several limitations. First, this study was designed as a retrospective review of data from a single institution. Because PALND was decided and performed by four surgeons, the indication for PALND was not standardized, which might have led to a selection bias. Second, as the number of patients with pathologic PALNM was small, the results of the multivariate analysis need to be interpreted with caution. Third, we 
Table 4 Prognostic factors for overall survival by univariate and multivariate analyses

\begin{tabular}{|c|c|c|c|c|c|}
\hline \multirow[t]{2}{*}{ Variables } & \multirow[t]{2}{*}{$n$} & \multicolumn{2}{|c|}{ Univariate analysis } & \multicolumn{2}{|l|}{ Multivariate analysis } \\
\hline & & 5-year OS (\%) & $p$ & HR (95\% Cl) & $p$ \\
\hline \multicolumn{6}{|c|}{ Pathologic T stage } \\
\hline T4 & 49 & 37.6 & $<0.001$ & $2.196(1.063-4.538)$ & 0.034 \\
\hline $\mathrm{T} 1-3$ & 214 & 82.1 & & & \\
\hline \multicolumn{6}{|c|}{ Pathologic N stage } \\
\hline $\mathrm{N} 1-2$ & 137 & 65.8 & 0.001 & $2.064(0.961-4.434)$ & 0.063 \\
\hline No & 126 & 87.7 & & & \\
\hline \multicolumn{6}{|l|}{ M stage } \\
\hline M1 & 32 & 38.8 & $<0.001$ & & \\
\hline MO & 231 & 81.5 & & & \\
\hline \multicolumn{6}{|c|}{ pathologic palnm } \\
\hline Positive & 19 & 54.0 & 0.002 & $1.367(0.505-3.703)$ & 0.538 \\
\hline Negative & 244 & 78.6 & & & \\
\hline \multicolumn{6}{|l|}{ RO resection } \\
\hline R2 & 20 & 12.8 & $<0.001$ & 4.643 (2.046-10.534) & $<0.001$ \\
\hline RO & 243 & 82.3 & & & \\
\hline
\end{tabular}

OS Overall survival; HR hazard ratio; CI Confidence interval; PALNM Paraaortic lymph node metastasis

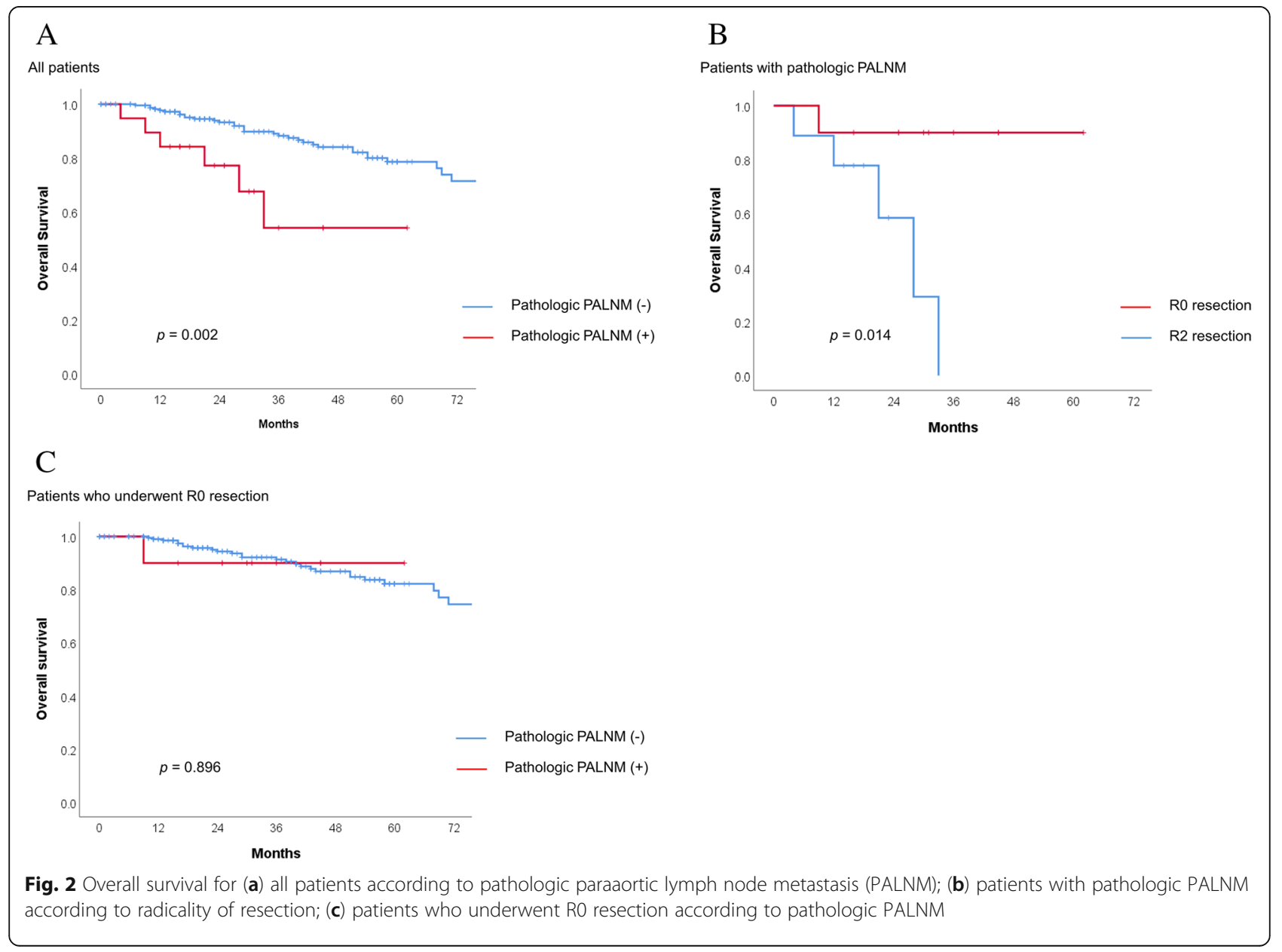


could not evaluate late complications such as ejaculatory dysfunction and reduced quality of life. Despite these limitations, the present study has a strength because very few studies have reported on the probability of pathologic PALNM in patients with incidentally detected PALNs.

\section{Conclusion}

The incidence of pathologic PALNM in patients with enlarged PALNs incidentally detected during colorectal cancer surgery was not negligible (2.2\%). If R0 resection can be achieved, patients with PALNM can show a relatively good prognosis. Therefore, we recommend performing PALND when an enlarged PALN is incidentally detected during surgery for the purpose of R0 resection.

\section{Abbreviations}

AJCC: American joint committee on cancer; CEA: Carcinoembryonic antigen; CT: Computed tomography; HR: Hazard ratio; OR: Odds ratio; OS: Overall survival; PALNs: Paraaortic lymph nodes; PALNM: Paraaortic lymph node metastasis; PALND: Paraaortic lymph node dissection; PET: Positron emission tomography

\section{Acknowledgements}

Not applicable

\section{Authors' contributions}

$J R L$ designed the study, analyzed the data, and wrote the manuscript. HM P, $\mathrm{CH} \mathrm{K}$, and HR K participated in the data collection. SY L participated in the study design and revised the manuscript. All authors have read and approved the final manuscript.

\section{Funding}

None

\section{Availability of data and materials}

The data that support the findings of this study are available from the corresponding author, upon reasonable request.

\section{Ethics approval and consent to participate}

This study was approved by the institutional review board of Chonnam National University Hwasun Hospital.

\section{Consent for publication}

Not applicable

\section{Competing interests}

The authors declare that they have no competing interests.

Received: 27 October 2020 Accepted: 28 December 2020 Published online: 12 January 2021

\section{References}

1. Ferlay J, Colombet M, Soerjomataram I, Mathers C, Parkin DM, Pineros M, et al. Estimating the global cancer incidence and mortality in 2018: GLOBOCAN sources and methods. Int J Cancer. 2019;144(8):1941-53.

2. Howlader N, Noone AM, Krapcho M, Miller D, Brest A, Yu M, et al. SEER Cancer Statistics Review, 1975-2017, National Cancer Institute. Bethesda, https://seer.cancer.gov/csr/1975_2017/, based on November 2019 SEER data submission, posted to the SEER web site, April 2020.

3. Amin MB, Greene FL, Edge SB, Compton CC, Gershenwald JE, Brookland RK, et al. The eighth edition AJCC cancer staging manual: continuing to build a bridge from a population-based to a more "personalized" approach to cancer staging. CA Cancer J Clin. 2017;67(2):93-9.

4. Min BS, Kim NK, Sohn SK, Cho CH, Lee KY, Baik SH. Isolated paraaortic lymph-node recurrence after the curative resection of colorectal carcinoma. J Surg Oncol. 2008:97(2):136-40.
5. Wong JS, Tan GH, Teo MC. Management of para-aortic lymph node metastasis in colorectal patients: a systemic review. Surg Oncol. 2016;25(4):411-8.

6. Choi PW, Kim HC, Kim AY, Jung SH, Yu CS, Kim JC. Extensive lymphadenectomy in colorectal cancer with isolated para-aortic lymph node metastasis below the level of renal vessels. J Surg Oncol. 2010;101(1):66-71.

7. Sahara K, Watanabe J, Ishibe A, Suwa Y, Suwa H, Ota M, et al. Long-term outcome and prognostic factors for patients with para-aortic lymph node dissection in left-sided colorectal cancer. Int J Colorectal Dis. 2019;34(6):1121-9,

8. Bae SU, Han YD, Cho MS, Hur H, Min BS, Baik SH, et al. Oncologic outcomes of colon cancer patients with extraregional lymph node metastasis: comparison of isolated paraaortic lymph node metastasis with resectable liver metastasis. Ann Surg Oncol. 2016;23(5):1562-8.

9. Kim CH, Lee SY, Kim HR, Kim YJ. Nomogram prediction of anastomotic leakage and determination of an effective surgical strategy for reducing anastomotic leakage after laparoscopic rectal cancer surgery. Gastroenterol Res Pract. 2017;2017:4510561.

10. Tsukamoto S, Kinugasa $Y$, Yamaguchi T, Shiomi A. Survival after resection of liver and lung colorectal metastases in the era of modern multidisciplinary therapy. Int J Colorectal Dis. 2014;29(1):81-7.

11. Saiura A, Yamamoto J, Hasegawa K, Koga R, Sakamoto Y, Hata S, et al. Liver resection for multiple colorectal liver metastases with surgery up-front approach: bi-institutional analysis of 736 consecutive cases. World I Surg. 2012;36(9):2171-8.

12. lida T, Nomori H, Shiba M, Nakajima J, Okumura S, Horio H, et al. Prognostic factors after pulmonary metastasectomy for colorectal cancer and rationale for determining surgical indications: a retrospective analysis. Ann Surg. 2013, 257(6):1059-64

13. Yamada K, Tsukamoto S, Ochiai H, Shida D, Kanemitsu Y. Improving selection for resection of synchronous para-aortic lymph node metastases in colorectal cancer. Dig Surg. 2019;36(5):369-75.

14. Nakai N, Yamaguchi T, Kinugasa Y, Shiomi A, Kagawa H, Yamakawa Y, et al. Long-term outcomes after resection of para-aortic lymph node metastasis from left-sided colon and rectal cancer. Int J Colorectal Dis. 2017;32(7):9991007.

15. Bae SU, Hur H, Min BS, Baik SH, Lee KY, Kim NK. Which patients with isolated para-aortic lymph node metastasis will truly benefit from extended lymph node dissection for colon cancer? Cancer Res Treat. 2018;50(3):712-9.

16. Ogura A, Akiyoshi T, Takatsu Y, Nagata J, Nagasaki T, Konishi T, et al. The significance of extended lymphadenectomy for colorectal cancer with isolated synchronous extraregional lymph node metastasis. Asian J Surg. 2017;40(4):254-61.

17. Gagniere J, Dupre A, Chabaud S, Peyrat P, Meeus P, Rivoire M. Retroperitoneal nodal metastases from colorectal cancer: curable metastases with radical retroperitoneal lymphadenectomy in selected patients. Eur J Surg Oncol. 2015;41(6):731-7.

18. Glass RE, Ritchie JK, Thompson HR, Mann CV. The results of surgical treatment of cancer of the rectum by radical resection and extended abdominoiliac lymphadenectomy. Br J Surg. 1985;72(8):599-601.

19. Arimoto A, Uehara K, Kato T, Nakamura H, Kamiya T, Nagino M. Clinical significance of para-aortic lymph node dissection for advanced or metastatic colorectal cancer in the current era of modern chemotherapy. Dig Surg. 2015;32(6):439-44.

20. Nakai N, Yamaguchi T, Kinugasa Y, Shiomi A, Kagawa H, Yamakawa Y, et al. Diagnostic value of computed tomography (CT) and positron emission tomography (PET) for paraaortic lymph node metastasis from left-sided colon and rectal cancer. Asian J Surg. 2020;43(6):676-82.

21. Cary C, Foster RS, Masterson TA. Complications of retroperitoneal lymph node dissection. Urol Clin North Am. 2019;46(3):429-37.

22. Tentes AA, Mirelis C, Karanikiotis C, Korakianitis O. Radical lymph node resection of the retroperitoneal area for left-sided colon cancer. Langenbecks Arch Surg. 2007:392(2):155-60.

23. Lee SY, Kim CH, Kim YJ, Kim HR. Impact of anal decompression on anastomotic leakage after low anterior resection for rectal cancer: a propensity score matching analysis. Langenbecks Arch Surg. 2015;400(7):791-6.

24. Kim CH, Kim HJ, Huh JW, Kim YJ, Kim HR. Learning curve of laparoscopic low anterior resection in terms of local recurrence. J Surg Oncol. 2014; 110(8):989-96.

\section{Publisher's Note}

Springer Nature remains neutral with regard to jurisdictional claims in published maps and institutional affiliations. 